\title{
Selected Topics on Advances in Capability-Oriented Information Systems Development: Editorial Introduction to Issue 10 of CSIMQ
}

\author{
Jelena Zdravkovic ${ }^{1 *}$, Óscar Pastor ${ }^{2}$ and Peri Loucopoulos ${ }^{3}$ \\ ${ }^{1}$ Stockholm University, Box 7003, Kista, 16407, Sweden \\ ${ }^{2}$ Universitat Politecnica de Valencia, Camí de Vera, s/n, 46022 València, Spain \\ ${ }^{3}$ CLMS Ltd, KD207, 4-6 University Way, London, UK \\ jelenaz@dsv.su.se (orcid.org/0000-0002-0870-0330), opastor@pros.upv.es \\ (orcid.org/0000-0002-1320-8471), periloucopoulos@icloud.com \\ (orcid.org/0000-0003-1395-5001)
}

Modern organizations need to be sustainable in the presence of dynamically changing business conditions, which require from Information Systems (IS) to address complex challenges for being able to support organizations acting in varying business conditions - changing customer demands, new legislations, new customers, and emerging alliances. From a technical perspective, the gap between business requirements and supporting IS is still present, mostly due to the fact that current IS development approaches operate with artifacts defined on a relatively low abstraction level. To go beyond the state of the art, IS development frameworks used by enterprises need to be structured for solving emerging problems, and enterprises need to have efficient methods for the use of these frameworks to deliver the right IS solutions just-in-time and just-enough. The notion of capability emerged in the beginning of the nineties in the context of competence-based management, military frameworks, and developing organization's competitive advantage - linguistically, it means the ability or qualities necessary to do something.

The objective of this thematic issue is to bring attention on emerging research in capabilityoriented business-IT alignment and systems' design. The five included articles origin from the best papers from the Conference on Advanced IS Engineering (CAiSE, http://caise.org) Workshop ASDENCA “Advances in Service DEsign Based on the Notion of CApability" in the period 2014-2016.

The thematic issue collection opens with the article entitled "A Comparative Analysis of Using the Capability Notion for Congruent Business- and Information System-Engineering" which analyzes how the notion of capability is included in the frameworks spanning from businessoriented, such as Business Architecture and Business Value Modeling, to the alignment-oriented frameworks represented by Enterprise Architecture (EA) and Enterprise Modeling (EM). The results of the analysis have shown that capability has widespread presence in the frameworks and that its conceptual meaning is largely similar, while the intentions and the mechanisms of its use differ raising stimulating opportunities for new contributions and improvements in the field.

\footnotetext{
${ }^{*}$ Corresponding author
}

(C) 2017 Jelena Zdravkovic et al. This is an open access article licensed under the Creative Commons Attribution License (http://creativecommons.org/licenses/by/4.0).

Reference: J. Zdravkovic, Ó. Pastor and P. Loucopoulos, "Selected Topics on Advances in CapabilityOriented Information Systems Development: Editorial Introduction to Issue 10 of CSIMQ," Complex Systems Informatics and Modeling Quarterly, CSIMQ, no. 10, pp. I-II, 2017. Available: https://doi.org/10.7250/csimq.2017-10.00 
The second article, "Value-Based and Context-Aware Selection of Software-Service Bundles: A Capability Based Method" addresses a well-recognized challenge for software vendors on how to optimize offering of different combinations of software products and services to different clients. Combinations, aka "bundles", of software products and associated service should be such as to best suit to client's individual requirements and circumstances. When circumstances change, the software-service bundle or its configuration need adaptation. The article proposes a capability-oriented method helping clients to select the most appropriate software-service combination.

"Combining Tools to Design and Develop Software Support for Capabilities" is the third article aiming to examine how a capability-oriented method for IS development can be combined with model-driven development tools to leverage both novel capability-based functionally and the proven functionality of existing tools. The examination is based on a case study where an existing model-driven software development tool is enriched by a capability-oriented approach.

The fourth article "LightCDD: Application of a Capability-Driven Development Method for Start-ups Development" proposes a lightweight capability-oriented enterprise modeling method to help entrepreneurs in the analysis, design, and specification of start-ups that are context-aware and adaptive to contextual changes and evolution. The method reduces the set of modeling constructs and guidelines from another, highly comprehensive capability-driven IS development methodology, to facilitate its adoption by entrepreneurs yet keeping it expressive enough for their purposes as well as compatible with the core methodology.

The Issue 10 of CSIMQ closes with the article "Capability-Driven Design of Business Service Ecosystem to Support Risk Governance in Regulatory Ecosystems" that examines how to raise the enterprise risk management from the enterprise level up to the ecosystem level by taking a capability-oriented approach as the theoretical foundation. To achieve the goal, the authors extend the Business Service Ecosystem (BSE) meta-model with the concepts from the Capability as a Service (CaaS) theory which is afterwards mapped with an information system risk management model to support risk governance at the ecosystem level. This mapping is illustrated and validated on the basis of an application case from the financial sector with respect to the most important concepts from the BSE: capability, resource, service, and goal.

For this special issue we owe thanks to the members of CSIMQ's Editorial Review Board for providing valuable reviews for the submitted articles. Special thanks go to the Managing Editor of the CSIMQ - Prof. Marite Kirikova for her great support in realizing this special issue, as well as to the publicizing team of the CSIMQ journal for their professional help and efficiency. Finally, we are grateful to the authors for submitting the articles reporting their scientifically innovate and passionate work.

To all readers - we hope you will enjoy in this issue and use it to conceive novel ideas in the area of capability-oriented business planning, enterprise modeling, IS design, and management. 